\title{
Well-posedness and attractors of the multi-dimensional hyperviscous magnetohydrodynamics equations
}

\author{
Hui Liu ${ }^{1}$ and Chengfeng Sun ${ }^{2}$ \\ ${ }^{1}$ Qufu Normal University \\ ${ }^{2}$ Nanjing University of Finance and Economics
}

May 5, 2020

\begin{abstract}
The multi-dimensional hyperviscous magnetohydrodynamics equation is considered in this paper. The well-posedness of the multi-dimensional hyperviscous magnetohydrodynamics equation is proved. Global attractor of the multi-dimensional hyperviscous magnetohydrodynamics equations is proved in $\mathrm{H}^{1 / 2+\mathrm{n} / 4} \times \mathrm{H}^{1 / 2+\mathrm{n} / 4}$ and $\mathrm{H}^{1+\mathrm{n} / 2} \times \mathrm{H}^{1+\mathrm{n} / 2}$.
\end{abstract}

\section{Hosted file}

Well-posedness-and-attractor-for-3D-hyperviscous-.pdf available at https://authorea.com/ users/300158/articles/429853-well-posedness-and-attractors-of-the-multi-dimensionalhyperviscous-magnetohydrodynamics-equations 